\title{
NOTES ON LINEARLY COMPACT ALGEBRAS
}

\section{TI YEN}

Let $A$ be a linearly compact ring with ideal neighborhoods of zero, and let $N$ be its radical. Zelinsky shows that [3, Theorem 1] $A / N$ is algebraically and topologically isomorphic to a complete direct sum (i.e., a cartesian product) of discrete simple rings with minimum condition. In case $A$ is commutative, then [3, Theorem 2] $A$ is algebraically and topologically isomorphic to a complete direct sum of a radical ring and primary rings with units, all the summands being linearly compact. If $A$ is an algebra and the closure of powers of $N$ has zero intersection, he then shows [4, Theorem C, p. 320] that $A$ (having the usual properties) satisfies the Wedderburn principal theorem. The restriction of $N$ is needed at two stages: raising of orthogonal idempotents of $A / N$ to orthogonal idempotents of $A$, and the inductive process of producing the semi-simple part. We propose to show that, if $A$ is commutative, the Wedderburn principal theorem is valid without restriction on $N$. The problem of raising orthogonal idempotents no longer exists, for idempotents which are orthogonal modulo $N$ are already orthogonal; indeed to each idempotent in $A / N$ there is only one idempotent representative in $A$. By [3, Theorem 2] we can restrict ourselves to primary algebras. Then $A / N$ is a field and we may avail ourselves of the results of field theory to construct the semi-simple part. Our main tool (Lemma 1) is a result of Jacobson [2, Theorem 6]. It also follows easily from this that we can raise a countable number of idempotents with no restriction on the radical. We use the terminology of [3].

The author wishes to acknowledge his thanks to Professor Zelinsky for his many valuable comments.

1. We need the following result which is a slight modification of [2, Theorem 6] to topological rings with ideal neighborhoods of zero. Its proof is effectively the same as that of [2, Theorem 6].

Lemma 1. Let $A$ be a topological ring with ideal neighborhoods of zero. Let $I$ be a closed subring contained in the radical of $A$. Then, for each $a \in I, a=0$ if the closure of $a I$ is $I$.

Let $A$ be a linearly compact commutative primary algebra with

Received by the editors November 19, 1954 and, in revised form, November 10 , 1955. 
unit over a field $K$. Then $A / N$ is a field extension of $K$. Every element $u$ not in the radical has an inverse, for it has an inverse $v$ modulo $N$ and $u v$ has an inverse by the definition of the radical. We assume that $A / N$ is separable over $K$, i.e., there is a transcendence base $\left(\alpha_{i}\right)_{i \in I}$ such that $A / N$ is separably algebraic over $K\left(\left(\alpha_{i}\right)_{i \in I}\right)$.

THEOREM 1. Let $A$ be a linearly compact commutative primary algebra with unit over a field $K$, and let $N$ be its radical. Suppose $A / N$ is separable over $K$. Then $A=S \oplus N$, where $S$ is a closed subalgebra isomorphic to $A / N$.

Proof. Let $\left(\alpha_{i}\right)_{i \in I}$ be a transcendence base of $A / N$ over $K$, and let $\left(a_{i}\right)_{i \in I}$ be a set of representatives in $A$. Then no polynomials in $\left(a_{i}\right)_{i \in I}$ with coefficients in $K$ will take values in $N$. Hence, $A$ contains the field $F$ generated by $\left(a_{i}\right)_{i \in I}$ over $K$, and $A$ may be considered as an algebra over $F$. Therefore, we may assume that $A / N$ is separably algebraic over $K$.

First consider the case that $A / N$ is finite over $K$. Then $A / N=K(\theta)$ for some element $\theta \in A / N$. Let $a$ be a representative of $\theta$. Denote by $K[a]$ the polynomial ring in $a . K[a]$ will be the desired algebra if $f(a)=0$, where $f$ is the minimal polynomial of $\theta$. We find such an $a$ as follows. Consider the collection of all subvarieties $a+I$, where $a$ is a representative of $\theta$ and $I$ is a closed ideal containing $f(a)$. Partially order this collection by set inclusion: $a+I>a^{\prime}+I^{\prime}$ if and only if $a+I \subset a^{\prime}+I^{\prime}$. Because of linear compactness, every linearly ordered subset of this collection has a least upper bound. Hence there is a maximal element, $a+I$ say. We claim $f(a)=0$. Suppose $f(a)=n \neq 0$. Since $A / N$ is separably algebraic over $K, f^{\prime}(a) \not \equiv 0(\bmod N)$, where $f^{\prime}$ denotes the formal derivative of $f$. Let $b$ be the inverse of $f^{\prime}(a)$ and $a^{\prime}=a-b n$. Then $f\left(a^{\prime}\right)=f(a-b n) \equiv f(a)-f^{\prime}(a) b n \equiv 0(\bmod n I)$. By Lemma $1, a^{\prime}+I^{\prime}>a+I$, where $I^{\prime}$ is the closure of $n I$. This contradicts the maximality of $a+I$.

In general, let $S$ be a maximal subfield contained in $A$. If $A / N \neq S$ then we may extend $S$ to a field $S(a)$ by above paragraph. Hence $A=S \oplus N$. Since $N$ is the unique maximal ideal of $A, S$ is a closed subalgebra of $A$.

REMARKs. (1) The subfield $S$ is unique if it is algebraic over $K .^{1}$ Suppose $A=S \oplus N=S^{\prime} \oplus N$. Take an $s \in S, s=s^{\prime}+n$ with $s^{\prime} \in S^{\prime}$ and $n \in N$. Let $f$ be the minimal polynomial of $s+N=s^{\prime}+N$. Then $0=f(s)=f\left(s^{\prime}+n\right)=f\left(s^{\prime}\right)+f^{\prime}\left(s^{\prime}\right) n+\cdots$. Since $f\left(s^{\prime}\right)=0$ and $f^{\prime}\left(s^{\prime}\right) \neq 0$, $n=0$ by Lemma 1 . It is clear that if $A / N$ is not algebraic over $K$ then $S$ is no longer unique.

1 This was suggested to us by Professor Zelinsky. 
(2) If $A$ is a primary ring with unit and of characteristic zero (i.e., $k x=0$ implies $x=0$, where $k$ is any positive integer), then Theorem 1 is still valid. For in this case $A$ can be considered as an algebra over the field of rational numbers.

(3) If $A / N$ is finite over $K$, the commutativity of $A$ enters only in $A / N$; when we deal with $A$ we only consider elements of the form $a, f(a), f^{\prime}(a), \cdots$. If only the commutativity of $A / N$ is assumed, we do not know whether $S$ is still unique.

Theorem 1 together with [3, Theorem 2] yields:

THEOREM 2. Let $A$ be a linearly compact commutative algebra over a field $K$, with ideal neighborhoods of zero. Then [3, Theorem 2] $A$ is algebraically and topologically a complete direct sum of primary algebras with unit and a radical algebra, each summand being linearly compact. Suppose that the quotient algebra of each primary summand over its radical is separable over $K$. Then A contains a closed subalgebra $S$ such that $A=S \oplus N$ (vector space direct sum), where $N$ is the radical of $A$. Moreover, $S$ is unique if each quotient algebra is algebraic over $K$.

2. The condition that the intersection of powers of $N$ be zero enters into the task of raising idempotents only when we want to raise any number of them. If we are willing to restrict ourselves to a countable number of idempotents then linear compactness alone will do, as is shown in the following lemma. From this we get an analogue of $[1$, Theorem 1].

Lemma 2. Let $A$ be a linear compact ring with ideal neighborhoods of zero, and $N$ its radical. Then a countable number of orthogonal idempotents can be raised to orthogonal idempotents in $A$.

Proof. It suffices to consider two idempotents $\bar{e}, \bar{f}$ in $A / N, \bar{e} \bar{f}=0$. Let $e$ be an idempotent in $A$ representing $\bar{e}$. If $a$ is a representative of $\bar{f}$, then $b=a-e a-a e+e a e$ is also a representative, and $e b=b e=0$. Let $I$ be the closed principal right ideal generated by $n=b^{2}-b$. Since $e b=0, e I=0$. Consider the collection of subvarieties $a+I$, where $a+N=\bar{f}, e a=a e=0$ and $I$ is the closed principal right ideal generated by $a^{2}-a$. Partially order this collection by set inclusion. By linear compactness there is a maximal element, $f+I$ say. Suppose $f^{2}-f \neq 0$. It follows from $(1-2 f)^{2}=1+4\left(f^{2}-f\right) \equiv 1(\bmod N)$, that $(1-2 f)$ has an inverse. ${ }^{2}$ Let $n=\left(f^{2}-f\right)(1-2 f)^{-1}, n \in I$, and let $I^{\prime}$ be the closure of $n I$. Then $f^{\prime}+I^{\prime}>f+I$, where $f^{\prime}=f+n$. This contradicts the maximality of $f+I$.

${ }^{2}$ The formal use of 1 is permissible, since it appears only in products with elements of $A$. 
TheORem 3. Let $A$ be a linearly compact algebra over a field $K$ with ideal neighborhoods of zero, and let $N$ be its radical. Then [3, Theorem 1] $A / N$ is algebraically and topologically isomorphic to a complete direct sum of discrete simple rings with minimal condition. Suppose that the summands are countable in number and that each summand is a total matrix algebra over $K$. Then there is a closed subalgebra $S$ such that $A=S \oplus N$.

Proof. We need only consider one summand. Then we follow the proof of [4, Theorem C, p. 320] summing up the semi-simple parts to get the subalgebra $S$. Let, therefore, $A / N$ be a total matrix algebra over $K$ and $\bar{e}_{i j}(i, j=1,2, \cdots, n)$ be a set of matrix units of $A / N$. The theorem will be established if we can raise $\bar{e}_{i j}$ to a set of matrix units $e_{i j}$ of $A$. By Lemma 2 we can find all the diagonal elements $e_{i i}$. It remains to find $e_{i j}(i \neq j)$. It suffices to find all the $e_{i 1}$ matching a given set of representatives $e_{1 i}(i=2, \cdots, n)$, where $e_{1 i}=e_{11} e_{1 i}=e_{1 i} e_{i i}$. Then put $e_{i j}=e_{i 1} e_{1 j}$.

We construct, for instance, $e_{21}$ as follows. If $a$ is any representative of $\bar{e}_{21}$ such that $a=e_{22} a=a e_{11}$, and if $a e_{12}=e_{22}$ then $e_{12} a=e_{11}$, for $e_{11}-e_{12} a$ is an idempotent in $N$. Consider the collection $a+I$, where $a$ is a representative of $\bar{e}_{21}$ with $a=e_{22} a=a e_{11}$ and $I$ is a closed right ideal containing $e_{22}-a e_{12}$. Partially order this collection by set inclusion. By linear compactness there is a maximal element $e_{21}+I$. We wish to show $e_{21} e_{12}=e_{22}$. Suppose $e_{22}-e_{21} e_{12}=n \neq 0$. Then $e_{21}^{\prime}+(n I)^{-}>e_{21}+I$, where $e_{21}^{\prime}=2 e_{21}-e_{21} e_{12} e_{21}$, contradicting the maximality of $e_{21}+I$.

Let $S$ be the total matrix algebra generated by $e_{i j}$. Since $N$ is the unique maximal ideal, $S$ is closed. We have $A=S \oplus N$.

\section{BIBLIOGRAPHY}

1. C. Feldman, The Wedderburn principal theorem in Banach algebras, Proc. Amer. Math. Soc. vol. 2 (1951) pp. 771-777.

2. N. Jacobson, The radical and semi-simplicity for arbitrary rings, Amer. J. Math. vol. 67 (1945) pp. 300-320.

3. D. Zelinsky, Linear compact modules and rings, Amer. J. Math. vol. 75 (1953) pp. 79-90.

4. —, Raising idempotents, Duke Math. J. vol. 21 (1954) pp. 315-323.

ILLINOIS INSTITUTE OF TECHNOLOGY 